\title{
Cousin's Lemma
}

\author{
Roland Coghetto \\ Rue de la Brasserie 5 \\ 7100 La Louvière, Belgium
}

Summary. We formalize, in two different ways, that "the $n$-dimensional Euclidean metric space is a complete metric space" (version 1. with the results obtained in [13, 26], 25] and version 2., the results obtained in [13, 14, (registrations) [24]).

With the Cantor's theorem - in complete metric space (proof by Karol Pąk in [22]), we formalize "The Nested Intervals Theorem in 1-dimensional Euclidean metric space".

Pierre Cousin's proof in 1892 [18 the lemma, published in 1895 [9] states that:

"Soit, sur le plan YOX, une aire connexe $S$ limitée par un contour fermé simple ou complexe; on suppose qu'à chaque point de $S$ ou de son périmètre correspond un cercle, de rayon non nul, ayant ce point pour centre : il est alors toujours possible de subdiviser $S$ en régions, en nombre fini et assez petites pour que chacune d'elles soit complétement intérieure au cercle correspondant à un point convenablement choisi dans $S$ ou sur son périmètre."

(In the plane YOX let $S$ be a connected area bounded by a closed contour, simple or complex; one supposes that at each point of $S$ or its perimeter there is a circle, of non-zero radius, having this point as its centre; it is then always possible to subdivide $S$ into regions, finite in number and sufficiently small for each one of them to be entirely inside a circle corresponding to a suitably chosen point in $S$ or on its perimeter) 23 .

Cousin's Lemma, used in Henstock and Kurzweil integral [29] (generalized Riemann integral), state that: "for any gauge $\delta$, there exists at least one $\delta$-fine tagged partition". In the last section, we formalize this theorem. We use the suggestions given to the Cousin's Theorem p.11 in [5] and with notations: 4], 29], 19], 28] and [12].

MSC: 54D30 03B35

Keywords: Cousin's lemma; Cousin's theorem; nested intervals theorem 
MML identifier: COUSIN, version: 8.1.04 5.36.1267

\section{Preliminaries}

Now we state the proposition:

(1) Let us consider non empty, increasing finite sequences $p, q$ of elements of $\mathbb{R}$. Suppose $p(\operatorname{len} p)<q(1)$. Then $p^{\frown} q$ is a non empty, increasing finite sequence of elements of $\mathbb{R}$.

Let us consider real numbers $a, b$. Now we state the propositions:

(2) If $1<a$ and $0<b<1$, then $\log _{a} b<0$.

(3) If $1<a$ and $1<b$, then $0<\log _{a} b$.

Let us consider a finite sequence $p$ and a natural number $i$.

Let us assume that $i \in \operatorname{dom} p$. Now we state the propositions:

(4) (i) $i=1$, or

(ii) $1<i$.

(5) (i) $i=\operatorname{len} p$, or

(ii) $i<\operatorname{len} p$.

Now we state the propositions:

(6) Let us consider an object $x$. Then $\prod\langle\{x\}\rangle=\{\langle x\rangle\}$.

(7) Let us consider an element $x$ of $\mathcal{R}^{1}$. Then there exists a real number $r_{3}$ such that $x=\left\langle r_{3}\right\rangle$.

(8) Let us consider a real number $a$. Then $\langle a\rangle$ is a point of $\mathcal{E}^{1}$.

(9) Let us consider real numbers $a, b$. If $a \leqslant b$, then $a \leqslant \frac{a+b}{2} \leqslant b$.

(10) Let us consider real numbers $a, b$, If $a \leqslant b<c$, then $a<\frac{b+c}{2}$.

Let us consider real numbers $a, b$. Now we state the propositions:

(11) If $a<b$, then $\frac{a+b}{2}<b$.

(12) If $a \leqslant b$, then $[a, b]$ is a non empty, compact subset of $\mathbb{R}$.

(13) Let us consider a finite sequence $f$. Suppose $2 \leqslant \operatorname{len} f$.

Then $f_{\lfloor 1}\left(\operatorname{len} f_{l 1}\right)=f(\operatorname{len} f)$. 


\section{2. $\mathcal{E}^{n}$ Is Complete - Proof Version 1}

From now on $n$ denotes a natural number, $s_{1}$ denotes a sequence of $\mathcal{E}^{n}$, and $s_{2}$ denotes a sequence of $\left\langle\mathcal{E}^{n},\|\cdot\|\right\rangle$.

Now we state the propositions:

(14) Let us consider elements $x, y$ of $\mathcal{E}^{n}$, and points $g, h$ of $\left\langle\mathcal{E}^{n},\|\cdot\|\right\rangle$. If $x=g$ and $y=h$, then $\rho(x, y)=\|g-h\|$.

(15) (i) $s_{1}$ is a sequence of $\left\langle\mathcal{E}^{n},\|\cdot\|\right\rangle$, and

(ii) $s_{2}$ is a sequence of $\mathcal{E}^{n}$.

Proof: $s_{1}$ is a sequence of $\left\langle\mathcal{E}^{n},\|\cdot\|\right\rangle$ by [10, (67), (22)]. $s_{2}$ is a sequence of $\mathcal{E}^{n}$ by [10, (22), (67)].

Let us assume that $s_{1}=s_{2}$. Now we state the propositions:

(16) $s_{1}$ is Cauchy if and only if $s_{2}$ is Cauchy sequence by norm. The theorem is a consequence of (14).

(17) $s_{1}$ is convergent if and only if $s_{2}$ is convergent. The theorem is a consequence of (14).

(18) Let us consider a sequence $S_{1}$ of $\mathcal{E}^{n}$. If $S_{1}$ is Cauchy, then $S_{1}$ is convergent. The theorem is a consequence of (15), (16), and (17).

(19) $\mathcal{E}^{n}$ is complete.

\section{3. $\mathcal{E}^{n}$ is Complete - Proof Version 2}

Now we state the propositions:

(20) The distance by norm of $\left\langle\mathcal{E}^{n},\|\cdot\|\right\rangle=\rho^{n}$. The theorem is a consequence of (14).

(21) MetricSpaceNorm $\left\langle\mathcal{E}^{n},\|\cdot\|\right\rangle=\mathcal{E}^{n}$. The theorem is a consequence of (20).

(22) $\mathcal{E}^{n}$ is complete. The theorem is a consequence of (21).

Let $n$ be a natural number. Let us note that $\mathcal{E}^{n}$ is complete.

\section{The Nested Intervals Theorem (1-Dimensional Euclidean Space)}

Let $a, b$ be sequences of real numbers. The functor IntervalSeq $(a, b)$ yielding a sequence of subsets of $\mathcal{R}^{1}$ is defined by

(Def. 1) for every natural number $i, i t(i)=\prod\langle[a(i), b(i)]\rangle$.

Now we state the propositions:

(23) Let us consider sequences $a, b$ of real numbers, and a natural number $i$. Then $(\operatorname{IntervalSeq}(a, b))(i)=\prod\langle[a(i), b(i)]\rangle$. 
(24) Let us consider sequences $a, b$ of real numbers. Then $\operatorname{IntervalSeq}(a, b)$ is a sequence of subsets of $\mathcal{E}^{1}$.

(25) $\prod\langle\mathbb{R}\rangle=\mathcal{R}^{1}$.

(26) Let us consider real numbers $a, b$, and points $x_{1}, x_{2}$ of $\mathcal{E}^{1}$. Suppose $x_{1}=\langle a\rangle$ and $x_{2}=\langle b\rangle$. Then $\rho\left(x_{1}, x_{2}\right)=|a-b|$.

(27) Let us consider real numbers $a, b$, and a subset $S$ of $\mathcal{E}^{1}$. Suppose $a \leqslant b$ and $S=\prod\langle[a, b]\rangle$. Let us consider points $x, y$ of $\mathcal{E}^{1}$. If $x, y \in S$, then $\rho(x, y) \leqslant b-a$.

Proof: Set $s=\prod\langle[a, b]\rangle$. For every points $x, y$ of $\mathcal{E}^{1}$ such that $x, y \in s$ holds $\rho(x, y) \leqslant b-a$ by $(6),[10$, (67), (22)], (7).

(28) Let us consider real numbers $a, b$, and a subset $S$ of $\mathcal{E}^{1}$. If $a \leqslant b$ and $S=\prod\langle[a, b]\rangle$, then $S$ is bounded.

Proof: Set $s=\prod\langle[a, b]\rangle$. There exists a real number $r$ such that $0<r$ and for every points $x, y$ of $\mathcal{E}^{1}$ such that $x, y \in s$ holds $\rho(x, y) \leqslant r$ by (6), [10, (67), (22)], (7).

Let us consider sequences $a, b$ of real numbers.

Let us assume that for every natural number $i, a(i) \leqslant b(i)$ and $a(i) \leqslant a(i+1)$ and $b(i+1) \leqslant b(i)$. Now we state the propositions:

(29) IntervalSeq $(a, b)$ is a non-empty, pointwise bounded, closed sequence of subsets of $\mathcal{E}^{1}$.

Proof: Reconsider $s=\operatorname{IntervalSeq}(a, b)$ as a sequence of subsets of $\mathcal{E}^{1}$. $s$ is non-empty by (23), [1, (26)], [3, (2)]. $s$ is pointwise bounded by (23), $(6),[10,(67),(22)] . s$ is closed by (23), [10, (67), (22)], (25).

(30) IntervalSeq $(a, b)$ is non ascending. The theorem is a consequence of (23).

(31) Let us consider real numbers $a, b, x$. If $a \leqslant x \leqslant b$, then $\langle x\rangle \in \prod\langle[a, b]\rangle$. Proof: Reconsider $P=\langle x\rangle$ as a point of $\mathcal{E}^{1}$. There exists a function $g$ such that $g=P$ and $\operatorname{dom} g=\operatorname{dom}\langle[a, b]\rangle$ and for every object $y$ such that $y \in \operatorname{dom}\langle[a, b]\rangle$ holds $g(y) \in\langle[a, b]\rangle(y)$ by [3, $(2)]$.

(32) Let us consider real numbers $a, b$, and a subset $S$ of $\mathcal{E}^{1}$. If $a \leqslant b$ and $S=\prod\langle[a, b]\rangle$, then $\varnothing S=b-a$. The theorem is a consequence of (28), $(31),(27),(8)$, and (26).

(33) Let us consider sequences $a, b$ of real numbers. Suppose for every natural number $i, a(i) \leqslant b(i)$ and $a$ is non-decreasing and $b$ is non-increasing. Then

(i) $a$ is convergent, and

(ii) $b$ is convergent.

(34) Let us consider sequences $a, b$ of real numbers. Suppose $a(0) \leqslant b(0)$ and for every natural number $i, a(i+1)=a(i)$ and $b(i+1)=\frac{a(i)+b(i)}{2}$ or 
$a(i+1)=\frac{a(i)+b(i)}{2}$ and $b(i+1)=b(i)$. Let us consider a natural number $i$. Then $a(i) \leqslant b(i)$.

Proof: Define $\mathcal{P}$ [object] $\equiv$ there exists a natural number $i$ such that $\$_{1}=i$ and $a(i) \leqslant b(i)$. For every natural number $k$ such that $\mathcal{P}[k]$ holds $\mathcal{P}[k+1]$. For every natural number $k, \mathcal{P}[k]$ from [2, Sch. 2].

Let us consider sequences $a, b$ of real numbers, a sequence $S$ of subsets of $\mathcal{E}^{1}$, and a natural number $i$. Now we state the propositions:

(35) Suppose $a(0) \leqslant b(0)$ and $S=\operatorname{IntervalSeq}(a, b)$ and for every natural number $i, a(i+1)=a(i)$ and $b(i+1)=\frac{a(i)+b(i)}{2}$ or $a(i+1)=\frac{a(i)+b(i)}{2}$ and $b(i+1)=b(i)$. Then

(i) $a(i) \leqslant b(i)$, and

(ii) $a(i) \leqslant a(i+1)$, and

(iii) $b(i+1) \leqslant b(i)$, and

(iv) $(\varnothing S)(i)=b(i)-a(i)$.

The theorem is a consequence of (34), (9), (24), (23), and (32).

(36) Suppose $a(0)=b(0)$ and $S=\operatorname{IntervalSeq}(a, b)$ and for every natural number $i, a(i+1)=a(i)$ and $b(i+1)=\frac{a(i)+b(i)}{2}$ or $a(i+1)=\frac{a(i)+b(i)}{2}$ and $b(i+1)=b(i)$. Then

(i) $a(i)=a(0)$, and

(ii) $b(i)=b(0)$, and

(iii) $(\varnothing S)(i)=0$.

Proof: Define $\mathcal{P}$ [natural number $] \equiv a\left(\$_{1}\right)=a(0)$ and $b\left(\$_{1}\right)=b(0)$. For every natural number $k$ such that $\mathcal{P}[k]$ holds $\mathcal{P}[k+1]$. For every natural number $k, \mathcal{P}[k]$ from [2, Sch. 2].

(37) Let us consider sequences $a, b$ of real numbers. Suppose for every natural number $i, a(i+1)=a(i)$ and $b(i+1)=\frac{a(i)+b(i)}{2}$ or $a(i+1)=\frac{a(i)+b(i)}{2}$ and $b(i+1)=b(i)$. Let us consider a natural number $i$, and a real number $r$. If $r=2^{i}$ and $r \neq 0$, then $b(i)-a(i) \leqslant \frac{b(0)-a(0)}{r}$.

Proof: Define $\mathcal{P}$ [object] $\equiv$ there exists a natural number $i$ and there exists a real number $r$ such that $\$_{1}=i$ and $r=2^{i}$ and $r \neq 0$ and $b(i)-a(i) \leqslant \frac{b(0)-a(0)}{r} . \mathcal{P}[0]$ by [17, (4)]. For every natural number $k$ such that $\mathcal{P}[k]$ holds $\mathcal{P}[k+1]$ by [17, (87), (6)]. For every natural number $k, \mathcal{P}[k]$ from [2, Sch. 2]. Consider $i_{1}$ being a natural number, $r_{1}$ being a real number such that $i=i_{1}$ and $r_{1}=2^{i_{1}}$ and $r_{1} \neq 0$ and $b\left(i_{1}\right)-a\left(i_{1}\right) \leqslant \frac{b(0)-a(0)}{r_{1}}$.

(38) Let us consider sequences $a, b$ of real numbers, and a sequence $S$ of subsets of $\mathcal{E}^{1}$. Suppose $a(0) \leqslant b(0)$ and $S=\operatorname{IntervalSeq}(a, b)$ and for every 
natural number $i, a(i+1)=a(i)$ and $b(i+1)=\frac{a(i)+b(i)}{2}$ or $a(i+1)=$ $\frac{a(i)+b(i)}{2}$ and $b(i+1)=b(i)$. Then

(i) $\varnothing S$ is convergent, and

(ii) $\lim \varnothing S=0$.

The theorem is a consequence of (36), (35), (34), (33), (3), and (37).

(39) Let us consider sequences $a, b$ of real numbers. Suppose $a(0) \leqslant b(0)$ and for every natural number $i, a(i+1)=a(i)$ and $b(i+1)=\frac{a(i)+b(i)}{2}$ or $a(i+1)=\frac{a(i)+b(i)}{2}$ and $b(i+1)=b(i)$. Then $\bigcap \operatorname{IntervalSeq}(a, b)$ is not empty. The theorem is a consequence of (24), (35), (29), (30), and (38).

(40) Let us consider a real number $r$, and sequences $a, b$ of real numbers. Suppose $0<r$ and $a(0) \leqslant b(0)$ and for every natural number $i, a(i+1)=$ $a(i)$ and $b(i+1)=\frac{a(i)+b(i)}{2}$ or $a(i+1)=\frac{a(i)+b(i)}{2}$ and $b(i+1)=b(i)$. Then there exists a real number $c$ such that

(i) for every natural number $j, a(j) \leqslant c \leqslant b(j)$, and

(ii) there exists a natural number $k$ such that $c-r<a(k)$ and $b(k)<c+r$. The theorem is a consequence of (39), (23), (24), (35), (29), and (38).

\section{Tagged Partition}

Now we state the propositions:

(41) Let us consider a non empty, closed interval subset $I$ of $\mathbb{R}$. Then there exist real numbers $a, b$ such that

(i) $a \leqslant b$, and

(ii) $I=[a, b]$.

(42) Let us consider non empty, closed interval subsets $I_{1}, I_{2}$ of $\mathbb{R}$. Suppose $\sup I_{1}=\inf I_{2}$. Then there exist real numbers $a, b, c$ such that

(i) $a \leqslant c \leqslant b$, and

(ii) $I_{1}=[a, c]$, and

(iii) $I_{2}=[c, b]$.

The theorem is a consequence of (41).

Let $A$ be a non empty, closed interval subset of $\mathbb{R}$ and $D$ be a partition of $A$. The set of tagged partitions of $D$ yielding a subset of $\mathbb{R}^{*}$ is defined by

(Def. 2) for every object $x, x \in$ it iff there exists a non empty, non-decreasing finite sequence $s$ of elements of $\mathbb{R}$ such that $x=s$ and $\operatorname{dom} s=\operatorname{dom} D$ and for every natural number $i$ such that $i \in \operatorname{dom} s \operatorname{holds} s(i) \in \operatorname{divset}(D, i)$. 
Now we state the propositions:

(43) Let us consider a non empty, closed interval subset $A$ of $\mathbb{R}$, and a partition $D$ of $A$. Then $D \in$ the set of tagged partitions of $D$.

Proof: For every natural number $i$ such that $i \in \operatorname{dom} D$ holds $D(i) \in$ $\operatorname{divset}(D, i)$ by $[15,(19)],(4)$.

(44) Let us consider real numbers $a, b$, and a non empty, closed interval subset $I_{4}$ of $\mathbb{R}$. If $I_{4}=[a, b]$, then $\langle b\rangle$ is a partition of $I_{4}$.

PRoOF: $\langle b\rangle$ is a partition of $I_{4}$ by [3, (39)], [15, (19)].

Let $I$ be a non empty, closed interval subset of $\mathbb{R}$ and $\varphi$ be a positive yielding function from $I$ into $\mathbb{R}$.

A tagged partition of $I$ and $\varphi$ is defined by

(Def. 3) there exists a partition $D$ of $I$ and there exists an element $T$ of the set of tagged partitions of $D$ such that it $=\langle D, T\rangle$.

Let $T_{1}$ be a tagged partition of $I$ and $\varphi$. We say that $T_{1}$ is $\delta$-fine if and only if

(Def. 4) there exists a partition $D$ of $I$ and there exists an element $T$ of the set of tagged partitions of $D$ such that $T_{1}=\langle D, T\rangle$ and for every natural number $i$ such that $i \in \operatorname{dom} D \operatorname{holds} \operatorname{vol}(\operatorname{divset}(D, i)) \leqslant \varphi(T(i))$.

\section{Partition Composition}

Let us consider a real number $r$. Now we state the propositions:

(45) (i) $\sup \{r\}=r$, and

(ii) $\inf \{r\}=r$.

(46) $\operatorname{vol}(\{r\})=0$. The theorem is a consequence of (45).

(47) Let us consider non empty, closed interval subsets $I_{1}, I_{2}$ of $\mathbb{R}$, and a positive yielding function $\varphi$ from $I_{1}$ into $\mathbb{R}$. Suppose $I_{2} \subseteq I_{1}$. Then $\varphi\left\lceil I_{2}\right.$ is a positive yielding function from $I_{2}$ into $\mathbb{R}$.

(48) Let us consider a non empty, closed interval subset $I$ of $\mathbb{R}$, and a real number $c$. Suppose $c \in I$. Then

(i) $[\inf I, c]$ is a non empty, closed interval subset of $\mathbb{R}$, and

(ii) $[c, \sup I]$ is a non empty, closed interval subset of $\mathbb{R}$, and

(iii) $\sup [\inf I, c]=\inf [c, \sup I]$.

The theorem is a consequence of (41).

Let $I_{5}, I_{6}$ be non empty, closed interval subsets of $\mathbb{R}, D_{4}$ be a partition of $I_{5}$, and $D_{6}$ be a partition of $I_{6}$. Assume $\sup I_{5} \leqslant \inf I_{6}$. The functor $D_{4} \cdot D_{6}$ 
yielding a non empty, increasing finite sequence of elements of $\mathbb{R}$ is defined by the term

(Def. 5) $\begin{cases}D_{4} \frown D_{6}, & \text { if } D_{6}(1) \neq \sup I_{5}, \\ D_{4} \frown D_{6}, 1, & \text { otherwise. }\end{cases}$

Now we state the propositions:

(49) Let us consider non empty, closed interval subsets $I_{5}, I_{6}$ of $\mathbb{R}$, a partition $D_{4}$ of $I_{5}$, and a partition $D_{6}$ of $I_{6}$. Suppose $\sup I_{5}=\inf I_{6}$ and len $D_{6}=1$ and $D_{6}(1)=\inf I_{6}$. Then $D_{4} \cdot D_{6}=D_{4}$.

(50) Let us consider non empty, closed interval subsets $I_{1}, I_{2}, I$ of $\mathbb{R}$. Suppose $\sup I_{1} \leqslant \inf I_{2}$ and $\inf I \leqslant \inf I_{1}$ and $\sup I_{2} \leqslant \sup I$. Then $I_{1} \cup I_{2} \subseteq I$.

(51) Let us consider non empty, closed interval subsets $I_{1}, I_{2}, I$ of $\mathbb{R}$, a partition $D_{1}$ of $I_{1}$, and a partition $D_{2}$ of $I_{2}$. Suppose $\sup I_{1} \leqslant \inf I_{2}$ and $I=\left[\inf I_{1}, \sup I_{2}\right]$. Then $D_{1} \cdot D_{2}$ is a partition of $I$. The theorem is a consequence of $(50)$.

(52) Let us consider a non empty, closed interval subset $I$ of $\mathbb{R}$, and a partition $D$ of $I$. Then the set of tagged partitions of $D$ is not empty.

(53) Let us consider a non empty, increasing finite sequence $s$ of elements of $\mathbb{R}$, and a real number $r$. Suppose $s($ len $s)<r$. Then $s^{\frown}\langle r\rangle$ is a non empty, increasing finite sequence of elements of $\mathbb{R}$. The theorem is a consequence of (1).

(54) Let us consider non empty, increasing finite sequences $s_{1}, s_{2}$ of elements of $\mathbb{R}$, and a real number $r$. Suppose $s_{1}\left(\right.$ len $\left.s_{1}\right)<r<s_{2}(1)$. Then $\left(s_{1} \frown\right.$ $\langle r\rangle)^{\frown} s_{2}$ is a non empty, increasing finite sequence of elements of $\mathbb{R}$. The theorem is a consequence of (53) and (1).

(55) Let us consider non empty, closed interval subsets $I_{1}, I_{2}, I$ of $\mathbb{R}$. Suppose $\sup I_{1}=\inf I_{2}$ and $I=I_{1} \cup I_{2}$. Then

(i) $\inf I=\inf I_{1}$, and

(ii) $\sup I=\sup I_{2}$.

(56) Let us consider a non empty, closed interval subset $I$ of $\mathbb{R}$, and a partition $D$ of $I$. Then

(i) $\operatorname{divset}(D, 1)=[\inf I, D(1)]$, and

(ii) for every natural number $j$ such that $j \in \operatorname{dom} D$ and $j \neq 1$ holds $\operatorname{divset}(D, j)=[D(j-1), D(j)]$.

Proof: For every natural number $j$ such that $j \in \operatorname{dom} D$ and $j \neq 1$ holds $\operatorname{divset}(D, j)=[D(j-1), D(j)]$ by [12, (4)].

(57) Let us consider a real number $r$, and finite sequences $p, q$ of elements of $\mathbb{R}$. Then len $\left(\left(p^{\frown}\langle r\rangle\right)^{\frown} q\right)=\operatorname{len} p+\operatorname{len} q+1$. 
(58) Let us consider a non empty, closed interval subset $I$ of $\mathbb{R}$, and a partition $D$ of $I$. Then every element of the set of tagged partitions of $D$ is not empty. The theorem is a consequence of (43).

(59) Let us consider a non empty, closed interval subset $I$ of $\mathbb{R}$, a partition $D$ of $I$, and an element $T$ of the set of tagged partitions of $D$. Then $\operatorname{rng} T \subseteq \mathbb{R}$. The theorem is a consequence of (43).

Let $I$ be a non empty, closed interval subset of $\mathbb{R}, \varphi$ be a positive yielding function from $I$ into $\mathbb{R}$, and $T_{1}$ be a tagged partition of $I$ and $\varphi$. The functor $T_{1}$-partition yielding a partition of $I$ is defined by

(Def. 6) there exists a partition $D$ of $I$ and there exists an element $T$ of the set of tagged partitions of $D$ such that $i t=D$ and $T_{1}=\langle D, T\rangle$.

\section{ExAmples of PARtitions}

In the sequel $r, s$ denote real numbers.

Now we state the proposition:

(60) Let us consider a function $\varphi$ from $[r, s]$ into $] 0,+\infty[$. Suppose $r \leqslant s$. Then the set of all $] x-\varphi(x), x+\varphi(x)[\cap[r, s]$ where $x$ is an element of $[r, s]$ is a family of subsets of $[r, s]_{\mathrm{T}}$.

Let us consider a function $\varphi$ from $[r, s]$ into $] 0,+\infty[$ and a family $S$ of subsets of $[r, s]_{\mathrm{T}}$.

Let us assume that $r \leqslant s$ and $S=$ the set of all $] x-\varphi(x), x+\varphi(x)[\cap$ $[r, s]$ where $x$ is an element of $[r, s]$. Now we state the propositions:

(61) $S$ is a cover of $[r, s]_{\mathrm{T}}$.

PROOF: $[r, s] \subseteq \bigcup S$ by [8, (3)].

(62) $S$ is open.

Proof: For every subset $P$ of $[r, s]_{\mathrm{T}}$ such that $P \in S$ holds $P$ is open by [11, (17)], [20, (35)], [11, (15), (9), (10)].

(63) Suppose $S=$ the set of all $] x-\varphi(x), x+\varphi(x)[\cap[r, s]$ where $x$ is an element of $[r, s]$. Then $S$ is connected.

Proof: For every subset $X$ of $[r, s]_{\mathrm{T}}$ such that $X \in S$ holds $X$ is connected by [16, (43)].

(64) Let us consider a function $\varphi$ from $[r, s]$ into $] 0,+\infty[$, and a family $S$ of subsets of $[r, s]_{\mathrm{T}}$. Suppose $r \leqslant s$ and $S=$ the set of all $] x-\varphi(x), x+\varphi(x)[\cap$ $[r, s]$ where $x$ is an element of $[r, s]$. Let us consider an interval cover $I$ of $S$. Then

(i) $I$ is a finite sequence of elements of $2^{\mathbb{R}}$, and

(ii) $\operatorname{rng} I \subseteq S$, and 
(iii) $\bigcup \operatorname{rng} I=[r, s]$, and

(iv) for every natural number $n$ such that $1 \leqslant n$ holds if $n \leqslant \operatorname{len} I$, then $I_{n}$ is not empty and if $n+1 \leqslant \operatorname{len} I$, then $\inf I_{n} \leqslant \inf I_{n+1}$ and $\sup I_{n} \leqslant \sup I_{n+1}$ and $\inf I_{n+1}<\sup I_{n}$ and if $n+2 \leqslant \operatorname{len} I$, then $\sup I_{n} \leqslant \inf I_{n+2}$, and

(v) if $[r, s] \in S$, then $I=\langle[r, s]\rangle$, and

(vi) if $[r, s] \notin S$, then there exists a real number $p$ such that $r<p \leqslant s$ and $I(1)=[r, p[$ and there exists a real number $p$ such that $r \leqslant p<s$ and $I($ len $I)=] p, s]$ and for every natural number $n$ such that $1<$ $n<$ len $I$ there exist real numbers $p, q$ such that $r \leqslant p<q \leqslant s$ and $I(n)=] p, q[$.

The theorem is a consequence of (61), (62), and (63).

(65) Let us consider real numbers $r, s, t, x$. Then

(i) if $r \leqslant x-t$ and $x+t \leqslant s$, then $] x-t, x+t[\cap[r, s]=] x-t, x+t[$, and

(ii) if $r \leqslant x-t$ and $s<x+t$, then $] x-t, x+t[\cap[r, s]=] x-t, s]$, and

(iii) if $x-t<r$ and $x+t \leqslant s$, then $] x-t, x+t[\cap[r, s]=[r, x+t$ [, and

(iv) if $x-t<r$ and $s<x+t$, then $] x-t, x+t[\cap[r, s]=[r, s]$.

(66) Let us consider real numbers $r, s, t, x$, and a subset $X_{1}$ of $\mathbb{R}$. Suppose $0<t$ and $r \leqslant x \leqslant s$ and $\left.X_{1}=\right] x-t, x+t[\cap[r, s]$. Then

(i) if $r \leqslant x-t$ and $x+t \leqslant s$, then $\inf X_{1}=x-t$ and $\sup X_{1}=x+t$, and

(ii) if $r \leqslant x-t$ and $s<x+t$, then $\inf X_{1}=x-t$ and $\sup X_{1}=s$, and

(iii) if $x-t<r$ and $x+t \leqslant s$, then $\inf X_{1}=r$ and $\sup X_{1}=x+t$, and

(iv) if $x-t<r$ and $s<x+t$, then $\inf X_{1}=r$ and $\sup X_{1}=s$.

The theorem is a consequence of (65).

Let us consider real numbers $a, b, c$, non empty, compact subsets $I_{5}, I_{6}$ of $\mathbb{R}$, a partition $D_{4}$ of $I_{5}$, a partition $D_{6}$ of $I_{6}$, and natural numbers $i, j$.

Let us assume that $a \leqslant c \leqslant b$ and $I_{5}=[a, c]$ and $I_{6}=[c, b]$. Now we state the propositions:

(67) Suppose $i \in \operatorname{dom} D_{4}$ and $j \in \operatorname{dom} D_{6}$. Then

(i) if $i<$ len $D_{4}$, then $D_{4}(i)<D_{6}(j)$, and

(ii) if $i=\operatorname{len} D_{4}$ and $c<D_{6}(1)$, then $D_{4}(i)<D_{6}(j)$, and

(iii) if $D_{6}(1)=c$, then $D_{4}\left(\right.$ len $\left.D_{4}\right)=D_{6}(1)$. 
Proof: If $i<\operatorname{len} D_{4}$, then $D_{4}(i)<D_{6}(j)$ by [3, (3)]. If $i=\operatorname{len} D_{4}$ and $c<D_{6}(1)$, then $D_{4}(i)<D_{6}(j)$ by [7, (6)], [3, (91)].

(68) If $i \in \operatorname{dom} D_{4}$ and $j \in \operatorname{dom} D_{6}$, then if $c<D_{6}(1)$, then $D_{4}(i)<D_{6}(j)$. The theorem is a consequence of (67).

(69) Let us consider real numbers $a, b, c$, and non empty, compact subsets $I_{4}$, $I_{5}, I_{6}$ of $\mathbb{R}$. Suppose $a \leqslant c \leqslant b$ and $I_{4}=[a, b]$ and $I_{5}=[a, c]$ and $I_{6}=[c, b]$. Let us consider a partition $D_{4}$ of $I_{5}$, and a partition $D_{6}$ of $I_{6}$. Suppose $c<D_{6}(1)$. Then $D_{4} \frown D_{6}$ is a partition of $I_{4}$.

Proof: Set $D_{5}=D_{4} \frown D_{6}$. For every extended reals $e_{1}, e_{2}$ such that $e_{1}$, $e_{2} \in \operatorname{dom} D_{5}$ and $e_{1}<e_{2}$ holds $D_{5}\left(e_{1}\right)<D_{5}\left(e_{2}\right)$ by [3, (25)], (68), [2, (11)], [3, (1)]. $\operatorname{rng} D_{5} \subseteq I_{4}$ by [3, (31)]. $D_{5}\left(\operatorname{len} D_{5}\right)=\sup I_{4}$ by [3, (3), (22)], [15, (19)].

(70) Let us consider real numbers $a, b$, and a non empty, closed interval subset $I_{4}$ of $\mathbb{R}$. Suppose $a \leqslant b$ and $I_{4}=[a, b]$. Let us consider a partition $D_{3}$ of $I_{4}$. If len $D_{3}=1$, then $D_{3}=\langle b\rangle$.

(71) Let us consider real numbers $a, b$, a non empty, compact subset $I_{4}$ of $\mathbb{R}$, and a partition $D_{3}$ of $I_{4}$. Suppose $2 \leqslant \operatorname{len} D_{3}$. Then $D_{3 \downarrow 1}$ is a partition of $I_{4}$.

Proof: Set $D=D_{3\lfloor 1}$. $D$ is a non empty, increasing finite sequence of elements of $\mathbb{R}$ by [3, (60)]. rng $D \subseteq I_{4}$ by [7, (33)]. $D(\operatorname{len} D)=\sup I_{4}$ by [3, (3)].

(72) Let us consider real numbers $a, b$. Suppose $a<b$. Then $\langle a, b\rangle$ is a non empty, increasing finite sequence of elements of $\mathbb{R}$.

Proof: Set $s=\langle a, b\rangle . s$ is increasing by [3, (44), (2)].

(73) Let us consider real numbers $a, b$, and a non empty, closed interval subset $I_{4}$ of $\mathbb{R}$. Suppose $a<b$ and $I_{4}=[a, b]$. Then $\langle a, b\rangle$ is a partition of $I_{4}$.

Proof: $\langle a, b\rangle$ is a partition of $I_{4}$ by $(72),[6,(127)],[3,(44)],[15,(19)]$.

\section{Cousin's Lemma}

Now we state the proposition:

(74) Let us consider real numbers $a, b$, and a positive yielding function $\varphi$ from $[a, b]$ into $\mathbb{R}$. Suppose $a \leqslant b$. Then there exists a non empty, increasing finite sequence $x$ of elements of $\mathbb{R}$ and there exists a non empty finite sequence $t$ of elements of $\mathbb{R}$ such that $x(1)=a$ and $x($ len $x)=b$ and $t(1)=a$ and $\operatorname{dom} x=\operatorname{dom} t$ and for every natural number $i$ such that $i-1, i \in \operatorname{dom} t$ holds $t(i)-\varphi(t(i)) \leqslant x(i-1) \leqslant t(i)$ and for every natural number $i$ such that $i \in \operatorname{dom} t$ holds $t(i) \leqslant x(i) \leqslant t(i)+\varphi(t(i))$. 
Proof: Define $\mathcal{P}$ [object] $\equiv$ there exists a non empty, increasing finite sequence $x$ of elements of $\mathbb{R}$ and there exists a non empty finite sequence $t$ of elements of $\mathbb{R}$ such that $x(1)=a$ and $x(\operatorname{len} x)=\$_{1}$ and $t(1)=a$ and $\operatorname{dom} x=\operatorname{dom} t$ and for every natural number $i$ such that $i-1, i \in \operatorname{dom} t$ holds $t(i)-\varphi(t(i)) \leqslant x(i-1) \leqslant t(i)$ and for every natural number $i$ such that $i \in \operatorname{dom} t$ holds $t(i) \leqslant x(i) \leqslant t(i)+\varphi(t(i))$. Consider $C$ being a set such that for every object $x, x \in C$ iff $x \in[a, b]$ and $\mathcal{P}[x]$. For every object $x$ such that $x \in C$ holds $x$ is real. Reconsider $c=\sup C$ as a real number. $c \in[a, b]$. Consider $d$ being an element of $\overline{\mathbb{R}}$ such that $d \in C$ and $c-\varphi(c)<d$. Consider $D_{0}$ being a non empty, increasing finite sequence of elements of $\mathbb{R}, T_{0}$ being a non empty finite sequence of elements of $\mathbb{R}$ such that $D_{0}(1)=a$ and $D_{0}\left(\operatorname{len} D_{0}\right)=d$ and $T_{0}(1)=a$ and $\operatorname{dom} D_{0}=\operatorname{dom} T_{0}$ and for every natural number $i$ such that $i-1$, $i \in \operatorname{dom} T_{0}$ holds $T_{0}(i)-\varphi\left(T_{0}(i)\right) \leqslant D_{0}(i-1) \leqslant T_{0}(i)$ and for every natural number $i$ such that $i \in \operatorname{dom} T_{0}$ holds $T_{0}(i) \leqslant D_{0}(i) \leqslant T_{0}(i)+\varphi\left(T_{0}(i)\right)$. $c \in C$ and $\mathcal{P}[c]$ by (1), [27, (32)], [3, (22), (39), (1)]. $c=b$ by (1), [27, $(32)],[3,(22),(39),(1)]$.

\section{Cousin's Lemma:}

Let us consider a non empty, closed interval subset $I$ of $\mathbb{R}$, and a positive yielding function $\varphi$ from $I$ into $\mathbb{R}$. Then there exists a tagged partition $T_{1}$ of $I$ and $\varphi$ such that $T_{1}$ is $\delta$-fine.

Proof: Consider $a, b$ being real numbers such that $a \leqslant b$ and $I=[a, b]$. Reconsider $r=\frac{1}{2}$ as a positive real number. Reconsider $\phi=r \cdot \varphi$ as a positive yielding function from $I$ into $\mathbb{R}$. Consider $x$ being a non empty, increasing finite sequence of elements of $\mathbb{R}, t$ being a non empty finite sequence of elements of $\mathbb{R}$ such that $x(1)=a$ and $x(\operatorname{len} x)=b$ and $t(1)=a$ and $\operatorname{dom} x=\operatorname{dom} t$ and for every natural number $i$ such that $i-1, i \in \operatorname{dom} t$ holds $t(i)-\phi(t(i)) \leqslant x(i-1) \leqslant t(i)$ and for every natural number $i$ such that $i \in \operatorname{dom} t$ holds $t(i) \leqslant x(i) \leqslant t(i)+\phi(t(i))$. Reconsider $D=x$ as a partition of $I$. Reconsider $T=t$ as an element of the set of tagged partitions of $D$. Reconsider $T_{1}=\langle D, T\rangle$ as a tagged partition of $I$ and $\varphi$. $T_{1}$ is $\delta$-fine by [15, (19)], (4), [8, (3)], [21, (20)].

\section{REFERENCES}

[1] Grzegorz Bancerek. König's theorem. Formalized Mathematics, 1(3):589-593, 1990.

[2] Grzegorz Bancerek. The fundamental properties of natural numbers Formalized Mathematics, 1(1):41-46, 1990.

[3] Grzegorz Bancerek and Krzysztof Hryniewiecki. Segments of natural numbers and finite sequences. Formalized Mathematics, 1(1):107-114, 1990.

[4] Robert G. Bartle. Return to the Riemann integral. American Mathematical Monthly, pages 625-632, 1996. 
[5] Robert G. Bartle. A modern theory of integration, volume 32. American Mathematical Society Providence, 2001.

[6] Czesław Byliński. Finite sequences and tuples of elements of a non-empty sets. Formalized Mathematics, 1(3):529-536, 1990.

[7] Czesław Byliński. Some properties of restrictions of finite sequences Formalized Mathematics, 5(2):241-245, 1996.

[8] Czesław Byliński. Functions and their basic properties Formalized Mathematics, 1(1): 55-65, 1990.

[9] Pierre Cousin. Sur les fonctions de $n$ variables complexes. Acta Mathematica, 19(1):1-61, 1895. doi $10.1007 /$ BFU2402869

[10] Agata Darmochwał. The Euclidean space. Formalized Mathematics, 2(4):599-603, 1991.

[11] Agata Darmochwał and Yatsuka Nakamura. Metric spaces as topological spaces - fundamental concepts Formalized Mathematics, 2(4):605-608, 1991.

[12] Noboru Endou and Artur Korniłowicz. The definition of the Riemann definite integral and some related lemmas Formalized Mathematıcs, 8(1):93-102, 1999.

[13] Noboru Endou and Yasunari Shidama. Completeness of the real Euclidean space. Formalized Mathematics, 13(4):577-580, 2005.

[14] Noboru Endou, Yasunari Shidama, and Katsumasa Okamura. Baire's category theorem and some spaces generated from real normed space. Formalized Mathematics, 14(4): 213-219, 2006. doi 10.2478/v10037-006-0024-x.

[15] Adam Grabowski and Yatsuka Nakamura. Some properties of real maps Formalized Mathematics, 6(4):455-459, 1997.

[16] Artur Korniłowicz. Properties of connected subsets of the real line. Formalized Mathematics, 13(2):315-323, 2005.

[17] Rafał Kwiatek. Factorial and Newton coefficients. Formalized Mathematics, 1(5):887-890, 1990.

[18] Bernard Maurey and Jean-Pierre Tacchi. La genèse du théorème de recouvrement de Borel Revue d'histoire des mathématiques, 11(2):163-204, 2005.

[19] Jean Mawhin. L'éternel retour des sommes de Riemann-Stieltjes dans l'évolution du calcul intégral. Bulletin de la Société Royale des Sciences de Liège, 70(4-6):345-364, 2001.

[20] Yatsuka Nakamura and Andrzej Trybulec. A decomposition of a simple closed curves and the order of their points. Formalized Mathematıcs, 6(4):563-572, 1997.

[21] Robin Nittka. Conway's games and some of their basic properties. Formalized Mathematics, 19(2):73-81, 2011. doi 10.2478/v10037-011-0013-6.

[22] Karol Pak. Complete spaces. Formalized Mathematics, 16(1):35-43, 2008. doi: $10.2478 /$ v10037-008-0006-2

[23] Manya Raman-Sundström. A pedagogical history of compactness The American Mathematical Monthly, 122(7):619-635, 2015.

[24] Hideki Sakurai, Hisayoshi Kunimune, and Yasunari Shidama. Uniform boundedness principle. Formalized Mathematics, 16(1):19-21, 2008. doi 10.2478/v10037-008-0003-5.

[25] Yasunari Shidama. Banach space of bounded linear operators Formalized Mathematics, 12(1):39-48, 2004.

[26] Yasumasa Suzuki, Noboru Endou, and Yasunari Shidama. Banach space of absolute summable real sequences Formalized Mathematics, 11(4):377-380, 2003.

[27] Wojciech A. Trybulec. Non-contiguous substrings and one-to-one finite sequences. Formalized Mathematics, 1(3):569-573, 1990.

[28] Lee Peng Yee. The integral à la Henstock. Scientiae Mathematicae Japonicae, 67(1): 13-21, 2008.

[29] Lee Peng Yee and Rudolf Vyborny. Integral: an easy approach after Kurzweil and Henstock, volume 14. Cambridge University Press, 2000.

Received December 31, 2015 